\title{
La economía social: concepto, macromagnitudes y yacimiento de empleo para el Trabajo Social
}

\author{
Social economy: concept, macromagnitudes and sources of employment \\ for the Social Work \\ Rafael CHAVEs ÁviLA \\ Universidad de Alcalá \\ rafael.chaves@uv.es
}

José Luis MonzÓn CAMPOS

Universidad de Valencia

ciriec@uv.es

Gustavo Zaragoza Pascual

Universidad de Valencia

gustavo.zaragoza@uv.es

Recibido: $21 / 10 / 2012$

Revisado: 16/11/2012

Aceptado: $25 / 02 / 2013$

Disponible on line: 16/04/2013

\begin{abstract}
Resumen
La economía social, el Tercer Sector de las economías situado entre el sector público y el sector privado tradicional, se encuentra en plena expansión en las dos últimas décadas, al calor de las grandes transformaciones sociales, económicas y de actuación de los poderes públicos. Tres son los objetivos de este artículo: en primer lugar, delimitar conceptualmente el campo de la economía social, recogiendo sus características estructurales e identificando entre sus actores principales a: las cooperativas, las mutualidades, las asociaciones con actividad económica, las fundaciones y ciertas entidades singulares como la ONCE. En segundo lugar, tras la precisa delimitación anterior, busca ofrecer las principales macromagnitudes de este sector y las familias que lo integran, tanto en España como en la Unión Europea, y puede concluirse al respecto que en España da empleo a cerca de 1.000 .000 personas y en la Unión Europea a unas 14.500.000, representando el 6,5 por ciento de la población ocupada. Finalmente, aborda las oportunidades que ofrece este campo para los trabajadores sociales desde la perspectiva del análisis de los cambios recientes en la normativa sobre servicios sociales. Palabras clave: economía social, tercer sector, magnitudes, empleo, España, Unión Europea.
\end{abstract}

\begin{abstract}
The Social Economy, a third sector between capitalist economy and public economy, is a field in expansion in modern societies in a context of economic and social transformations and of restructuring of public action.

The aims of this article are three. First, to conceptualize the field of the Social Economy and to identify its main structural agents. Second, to present general figures of this socioeconomic field in Spain and in Europe. The main conclusion is that the Social Economy is a very important in human and economic terms: over 14,5 million paid employees in Europe and near one million in Spain. Last, to analyze the opportunities of this field for social workers in the future considering recent changes in social services laws.
\end{abstract}

Keywords: social economy, third sector, figures, employment, Spain, European Union.

Referencia normalizada: Chaves Ávila, R., Monzón Campos, J. L., y Zaragoza Pascual, G. (2013): «La economía social: concepto, macromagnitudes y yacimiento de empleo para el Trabajo Social». Cuadernos de Trabajo Social, 26(1): 19-29.

Sumario: Introducción. 1. La delimitación conceptual de economía social. 2. La economía social en España. 3. La economía social en la Unión Europea. 4. La economía social como yacimiento de empleo para Trabajo Social.

5. Conclusiones. 6. Referencias bibliográficas.

\section{Introducción}

Es en contextos de crisis cuando las personas y los países se encuentran más predispuestos a buscar soluciones innovadoras a sus problemas, y más atentos a respuestas sociales que han demostrado una capacidad exitosa. Es éste 
el caso de la economía social, concebida como ámbito económico situado entre la economía pública y la economía privada tradicional. Históricamente ha emergido para dar respuestas sustantivas a los problemas de diversos agentes económicos y satisfacer demandas sociales. Es así como emergieron las sociedades de socorro mutuo en el siglo XIX como precursoras de la seguridad social, para cubrir colectivamente riesgos de carácter sociolaboral; como surgieron las cooperativas de consumo y de vivienda para dar respuesta a demandas de bienes de primera necesidad; las cooperativas de trabajo y las sociedades laborales; las empresas de inserción y los centros especiales de empleo, como formas organizativas diseñadas para solucionar problemas de empleo.

El conjunto de todo este ámbito económico diferenciado, de este Tercer Sector de las economías, ha recibido en los últimos decenios diferentes denominaciones y concepciones en la literatura científica y por los propios actores. La concepción que ha imperado en España y Europa ha sido la de la economía social, aunque ha coexistido con la de las entidades no lucrativas y con la de las empresas sociales.

El presente artículo persigue un triple objetivo: en primer lugar, ofrece una delimitación conceptual de la Economía Social, la de mayor arraigo en la literatura científica, y la pone en relación con las otras dos concepciones citadas e identifica, a continuación, a los actores que la integran. En segundo lugar, merced a los diferentes trabajos científicos realizados por la asociación Centro Internacional de Investigación e Información sobre la Economía pública, social y cooperativa (CIRIEC), ofrece las principales macromagnitudes de este sector y las familias que lo integran tanto para España como para la Unión Europea. Finalmente, aborda las oportunidades que este campo ofrece para los trabajadores sociales.

\section{La delimitación conceptual de economía social}

\subsection{La identificación de la economía social por sus protagonistas}

Desde finales de los años setenta, cuando las plataformas representativas francesas de las cooperativas, las mutualidades y las asociaciones se integraron en el Comité Nacional de
Liaison des Mutuelles, Coopératives et Associations (CNLMCA), han sido varias las autodefiniciones de Economía Social realizadas por las propias organizaciones representativas de este sector, tanto a nivel nacional como internacional. La primera definición, realizada por la citada entidad paraguas sirvió de eje para el despliegue de las primeras políticas públicas desarrolladas para este sector, ya en el año 1982, por el primer gobierno Mitterrand.

En la actualidad la entidad representativa de este sector socioeconómico a escala europea, la Social Economy Europe, concibe como características identitarias de la misma unos principios recogidos en la Carta de Principios de la Economía Social (junio, 2002) que son los siguientes:

- Primacía de la persona y del objeto social sobre el capital.

- Adhesión voluntaria y abierta.

- Control democrático por sus miembros.

- Conjunción de los intereses de los miembros y del interés general.

- Defensa y aplicación de los principios de solidaridad y responsabilidad.

- Autonomía de gestión e independencia respecto de los poderes públicos.

- Destino de la mayoría de los excedentes a la consecución de objetivos a favor del desarrollo sostenible, la mejora de los servicios a los miembros y el interés general.

\subsection{La emergente definición jurídica de economía social}

Las instituciones públicas no han sido ajenas a esta realidad social y económica. Por un lado han sido instituciones europeas, como el Comité Económico y Social Europeo en su Dictamen 01/10/2009 sobre «Diversidad de formas de empresa», y el Parlamento Europeo, Informe sobre Economía Social (26/01/2009), las que han recogido unas delimitaciones precisas de este campo. Por otro lado, han sido los gobiernos nacionales y regionales de la Unión Europea quienes han marcado jurídicamente los perfiles de la economía social. El primero ha sido el Decreto de la región belga de Valonia $(20 / 11 / 2008)$ al que le siguió la Ley 5/2011 de 29 de marzo, de economía social española. También Grecia ese mismo año promulgó la Ley 4019/2011 sobre Social Economy, Social 
Enterpreneurship and other provisions. En 2012 han sido promulgados dos nuevos decretos en las regiones belgas de Flandes y Bruselas, encontrándose en proyecto de ley en Portugal, Polonia, Rumanía y en Francia con el nuevo gobierno del Presidente Hollande, quien ha nombrado por primera vez en la historia institucional de Europa, a un Ministro para la economía social y solidaria.

En lo relativo a la Ley 5/2011 cabe destacar que la definición recogida enlaza con la tradición histórica europea de raíz francófona del concepto amplio de Economía Social, que incluye dos tipos de entidades: aquellas definidas jurídicamente, en particular, las cooperativas, las mutualidades, las asociaciones que lleven a cabo actividades económicas, las fundaciones, las sociedades laborales, las empresas de inserción, los centros especiales de empleo, las sociedades agrarias de transformación y las cofradías de pescadores, así como determinadas entidades singulares, creadas por normas específicas, como la Organización nacional de ciegos españoles (ONCE), que recoge la propia Disposición adicional tercera, y otras formas jurídicas no incluidas en las anteriores categorías, que cumplen los principios orientadores y que se encuentren incluidas en un catálogo de entidades elaborado por el Ministerio de Trabajo en colaboración con otros órganos públicos.

\subsection{La definición científica de economía social}

A pesar de la gran importancia que tienen las empresas de economía social, se poseen pocos datos fiables, precisos y comparables sobre las mismas, si bien se han producido notables avances en los últimos años. Dos son las principales razones que explican esta invisibilidad institucional de la economía social: en primer lugar, los dos sistemas de contabilidad nacional más importantes actualmente en vigor, el SCN-1993 de Naciones Unidas y el SEC-1995 de la Unión Europea, no reconocen como un sector institucional diferenciado, al conjunto de operadores económicos que integran la economía social. Sin embargo, recientemente la Comisión Europea ha editado un «Manual de orientación para la elaboración de una cuenta satélite de las empresas de Economía Social (cooperativas y mutuas)» (Monzón y Barea,
2007), lo que está permitiendo obtener datos armonizados y fiables de una parte muy significativa de dicha economía, constituida por cooperativas, mutuas y otras empresas similares.

Un hito en el proceso de delimitación conceptual ha sido el Manual de cuentas satélite de la Comisión Europea, que ha integrado en un único concepto los principios históricos y valores propios de la economía social y la metodología de los sistemas de contabilidad nacional en vigor (SEC-1995 y SCN-1993). A partir de los criterios establecidos por el Manual, el Informe Chaves-Monzón del Comité Económico y Social Europeo (Chaves y Monzón, 2008) ha formulado una definición operativa de economía social basada en un amplio consenso político y científico que está permitiendo cuantificar y hacer visible de forma homogénea y armonizada internacionalmente los principales datos agregados de las entidades que la integran. Dicha definición es:

Conjunto de empresas privadas organizadas formalmente, con autonomía de decisión y libertad de adhesión, creadas para satisfacer las necesidades de sus socios a través del mercado, produciendo bienes y servicios, asegurando o financiando y en las que la eventual distribución entre los socios de beneficios o excedentes así como la toma de decisiones, no están ligados directamente con el capital o cotizaciones aportados por cada socio, correspondiendo un voto a cada uno de ellos. La Economía Social también agrupa a aquellas entidades privadas organizadas formalmente con autonomía de decisión y libertad de adhesión que producen servicios de no mercado a favor de las familias, cuyos excedentes, si los hubiera, no pueden ser apropiados por los agentes económicos que las crean, controlan o financian».

Esta definición, congruente con la formulada por los propios actores de la economía social, configura dos grandes subsectores de la misma, el de productores de mercado y el de productores de no mercado, clasificación que es muy útil para la elaboración de estadísticas fiables y la realización de análisis de la actividad económica, de conformidad con los sistemas de contabilidad nacional, sin perjuicio de los estrechos vínculos existentes en la economía social entre el mercado y el no mercado, derivados de una característica común a todas 
sus organizaciones; a saber, que son entidades de personas que desarrollan una actividad económica con el objetivo prioritario de satisfacer necesidades de otras personas, antes que de retribuir a inversores capitalistas.

\subsection{Identificación de los diferentes actores incluidos en el concepto de economía social} Tanto el Manual de la Comisión Europa como el Informe Chaves-Monzón realizan un análisis pormenorizado de la delimitación conceptual de economía social que se ha realizado en el anterior epígrafe e identifican a sus principales actores, agrupándolos en los dos grandes subsectores: de mercado y de no-mercado. Las características comunes para ambos subsectores son: entidades privadas, organizadas formalmente, con autonomía de decisión y libertad de adhesión, con procesos democráticos de decisión y en los que la eventual distribución de beneficios o excedentes, si se produce, no es proporcional al capital o a las cotizaciones aportadas por los socios, sino de acuerdo con la actividad que éstos realizan con la entidad (Chaves y Monzón, 2008).

\section{Subsector de mercado}

El subsector de mercado en la economía social (ES) está constituido fundamentalmente por cooperativas, mutuas y mutualidades de previsión social, y grupos empresariales controlados por entidades de la misma, y otras empresas similares como empresas de inserción y sociedades no financieras controladas mayoritariamente por trabajadores con procesos democráticos de decisión y distribución equitativa de sus beneficios. También pueden encontrarse en algunos países sociedades de ahorro y crédito y cajas de ahorros que se ajustan a la definición formulada de «empresas de economía social».

\section{Subsector de no-mercado}

Este subsector está constituido mayoritariamente por asociaciones y fundaciones, aunque también pueden encontrarse entidades con otra forma jurídica. Está integrado por todas aquellas entidades de economía social que, según los criterios establecidos por la contabilidad nacional, no son productores de mercado; es decir, aquellos cuya producción se suministra mayoritariamente de forma gratuita o a precios económicamente no significativos. Se trata de entidades privadas organizadas formalmente, con autonomía de decisión y libertad de adhesión que producen servicios de no mercado a favor de las familias, cuyos excedentes, si los hubiera, no pueden apropiárselo los agentes que las crean, controlan o financian. Es decir, se trata de entidades no lucrativas en sentido estricto, que aplican el principio de no distribución de beneficios (PNDB) y en las que, como en todas las entidades de economía social, los individuos son los verdaderos beneficiarios de los servicios producidos.

La mayoría de estas entidades tienen un funcionamiento democrático si bien pueden existir algunas que carezcan de estructuras democráticas. En este último caso, también se incluyen en la Economía Social a las entidades del denominado tercer sector de acción social, que suministran a las familias de forma gratuita bienes sociales o preferentes de indudable utilidad social.

\section{La economía social en España}

España constituye uno de los países europeos con mayor reconocimiento y despliegue socioeconómico de economía social. No es casualidad, por lo tanto, que haya sido el primer país del viejo continente que haya promulgado una ley de economía social de ámbito nacional.

En relación con la importancia económica de la economía social, los trabajos del propio Centro Internacional de Investigación e Información sobre la Economía pública social y cooperativa (CIRIEC), a lo largo de los años han perfilado cuantitativamente este sector. El último de sus informes, basado en un amplio trabajo de campo ofrece el siguiente dato: el valor añadido bruto de toda la economía social con relación al PIB era en 2008 del 2,47 por ciento, proporcionaba empleo directo a 1.243 .596 personas en 200.858 entidades y empresas (Monzón, 2010).

Cabe destacar que algo más de la mitad del total del empleo de la economía social, en concreto 643.828 personas, trabajaban en entidades de economía social donde los trabajadores sociales encuentran más posibilidades de empleo por su mayor vinculación con los servicios sociales y sociosanitarios: las asociaciones privadas al servicio de los hogares, las fundaciones, las entidades singulares como Cáritas, Cruz Roja y ONCE, las empresas de inserción y los centros especiales de empleo (Tabla 1). 


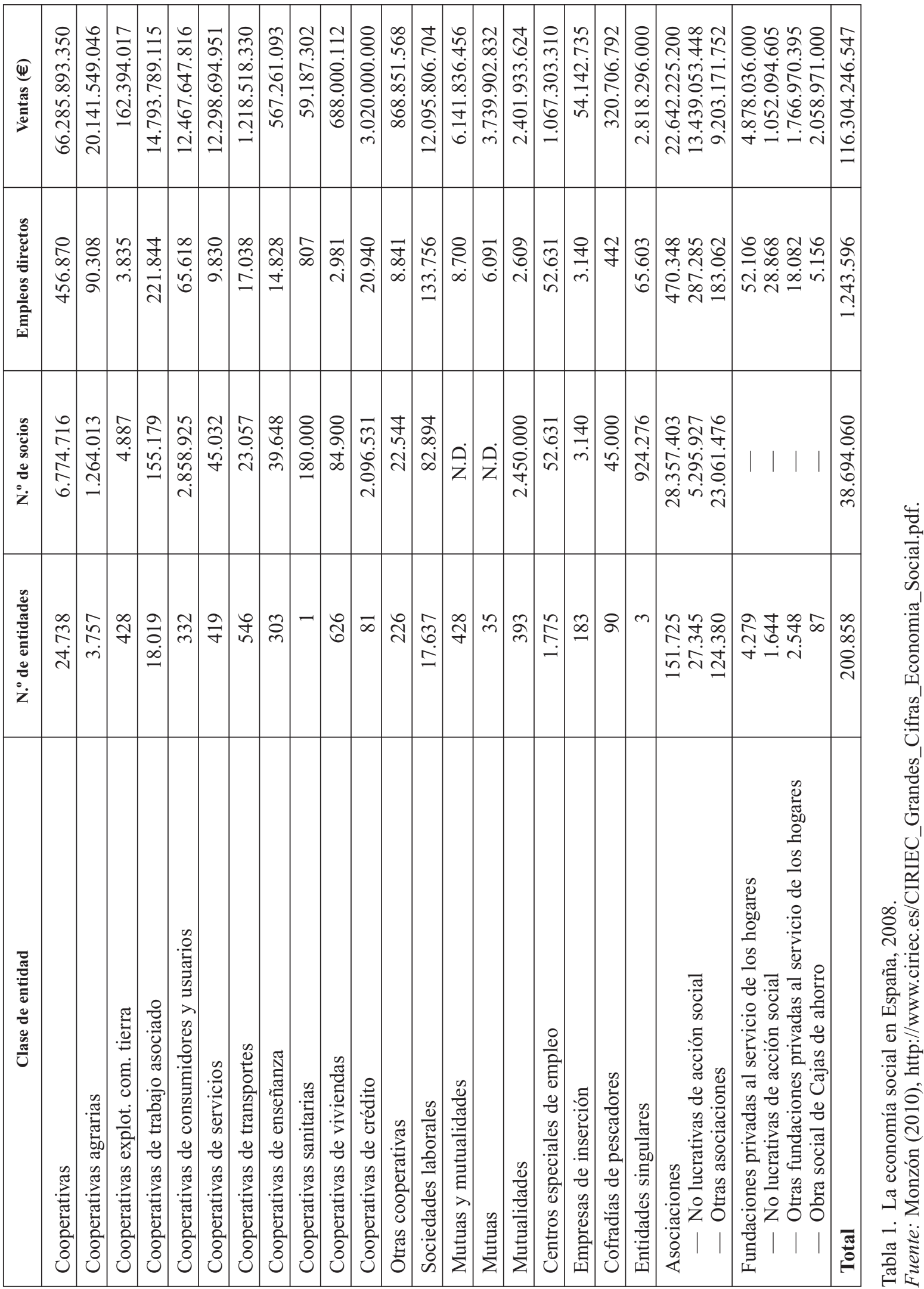




\section{La economía social en la Unión Europea}

La economía social europea constituye una amplia realidad, tanto en términos humanos como económicos. En el año 2009 daba empleo a 14.500 .000 personas, representando el 6,5 por ciento de la fuerza de trabajo de los 27 países de la Unión Europea. Esta fuerza laboral ha incrementado de modo sostenido a lo largo de la década pasada, pasando de dar empleo a 11.000 .000 personas en 2002-2003 a dárselo a 14.500 .000 personas 7 años después.

No obstante lo anterior, se aprecian importantes diferencias tanto entre los 15 países miembros más antiguos, donde tanto el incremento del empleo en este periodo como el porcentaje sobre la población laboral, presentan registros superiores a los de los nuevos países miembros de la Unión.

Otra importante conclusión, de especial relevancia en el contexto del presente artículo - donde se presta atención al potencial de empleo de la economía social para los trabajadores sociales - es que dos terceras partes de este empleo se mantiene en la «familia» de las asociaciones y afines (fundaciones y otras entidades sin fines lucro), habiendo experimentado este empleo también un incremento sostenido a lo largo de la década de 2000-2010, como se desprende de los datos de la Tabla 2.

La incidencia de la crisis actual ha sido notable también en la economía social, y de modo especial en la rama de las asociaciones y afines, ligado a los problemas de financiación del Estado de bienestar y a la transferencia de fondos a estas entidades sociales. A pesar de ello, en Italia el conjunto de la economía social ha continuado incrementando su nivel de ocupación en 2010 y en 2011 y las 106 cooperativas sociales mayores han pasado de 75.828 empleados en 2008, a 81.156 en 2009 y 84.243 en 2010. En el Reino Unido, el sector voluntario ha pasado de dar empleo a 642.000 personas en 2007, el 2,3 por ciento de la fuerza de trabajo británica a 765.000 personas en 2010 . Desde 2001 incrementó en un 40 por ciento su número de puestos de trabajo demostrando con ello su capacidad de generación de empleo. No obstante, la crisis financiera del Estado británico ha reducido drásticamente los fondos públicos destinados al sector voluntario para 2011 y 2012, incidiendo directamente sobre el nivel de empleo en este último, repitiendo situacio- nes ya vividas en los años 1980 en la era Thatcher y Reagan.

\section{La economía social como yacimiento de empleo para Trabajo Social}

Este último apartado tiene como finalidad analizar alguna de las oportunidades que se ofrecen al Trabajo Social como disciplina científica y profesional, desde la economía social. Hemos optado, en esta ocasión, por acotar el ámbito de interés al desarrollo y despliegue de las prestaciones y servicios propios del Sistema Público de Servicios Sociales, para lo cual hemos llevado a cabo un estudio de las últimas leyes de Servicios Sociales, destacando aquellos ámbitos de intervención propios del Trabajo Social e igualmente hemos identificado las referencias legislativas existentes, en dichas normas sobre el Tercer Sector y la iniciativa social.

Comenzaremos por una aproximación terminológica que contextualice los distintos conceptos utilizados. Es evidente que nos vamos a situar en el marco de lo que se conoce como política social, es decir «aquella política relativa a la administración pública de la asistencia, esto es al desarrollo y dirección de los servicios específicos del Estado y de las autoridades locales en aspectos tales como salud, educación trabajo, vivienda, asistencia y servicios sociales». Este enfoque se desarrolla a partir de T.H. Marshall (1998) que la define, también, como «la política de los gobiernos encaminada a tener un impacto directo en el bienestar de los ciudadanos a base de proporcionales servicios o ingresos.») La política social se concreta en un sistema jurídico e institucional de protección, previsión y asistencia de ciertas necesidades y oportunidades vitales determinadas por el orden social vigente. Conviene destacar, desde el primer momento, los lazos de convergencia existentes entre política social y Trabajo Social, especialmente debido a que una gran parte de las actuaciones profesionales de numerosos trabajadores sociales consiste en la traducción de las políticas sociales en un servicio operativo, gestionando prestaciones o servicios procedentes de los distintos sistemas de protección social. Por lo tanto podemos subrayar las numerosas convergencias existentes en ámbitos empíricos y también teóricos, por lo que se refiere a los primeros, al hacer llegar a los ciudadanos, lo legislado, tanto a individuos como a grupos poblacionales 


\begin{tabular}{|c|c|c|c|c|}
\hline País & Cooperativas & Mutualidades & Asociaciones & Total \\
\hline Austria & 61.999 & 1.416 & 170.113 & 233.528 \\
\hline Bélgica & 13.547 & 11.974 & 437.020 & 462.541 \\
\hline Dinamarca & 70.757 & 4.072 & 120.657 & 195.486 \\
\hline Finlandia & 94.100 & 8.500 & 84.600 & 187.200 \\
\hline Francia & 320.822 & 128.710 & 1.869 .012 & 2.318 .544 \\
\hline Alemania & 830.258 & 86.497 & 1.541 .829 & 2.458 .584 \\
\hline Grecia & 14.983 & 1.140 & 101.000 & 117.123 \\
\hline Irlanda & 43.328 & 650 & 54.757 & 98.735 \\
\hline Italia & 1.128 .381 & n.a. & 1.099 .629 & 2.228 .010 \\
\hline Luxemburgo & 1.933 & n.a. & 14.181 & 16.114 \\
\hline Portugal & 51.391 & 5.500 & 194.207 & 251.098 \\
\hline Países Bajos & 184.053 & 2.860 & 669.121 & 856.054 \\
\hline España & 646.397 & 8.700 & 588.056 & 1.243 .153 \\
\hline Suecia & 176.816 & 15.825 & 314.568 & 507.209 \\
\hline Reino Unido & 236.000 & 50.000 & 1.347 .000 & 1.633 .000 \\
\hline \multicolumn{5}{|l|}{ Nuevos países miembros } \\
\hline Bulgaria & 41.300 & n.a. & 80.000 & 121.300 \\
\hline Chipre & 5.067 & n.a. & n.a. & 5.067 \\
\hline República Checa & 58.178 & 5.679 & 96.229 & 160.086 \\
\hline Estonia & 9.850 & n.a. & 28.000 & 37.850 \\
\hline Hungría & 85.682 & 6.676 & 85.852 & 178.210 \\
\hline Letonia & 440 & n.a. & n.a. & 440 \\
\hline Lituania & 8.971 & n.a. & n.a. & 8.971 \\
\hline Malta & 250 & n.a. & 1.427 & 1.677 \\
\hline Polonia & 400.000 & 2.800 & 190.000 & 592.800 \\
\hline Rumania & 34.373 & 18.999 & 109.982 & 163.354 \\
\hline Eslovaquia & 26.090 & 2.158 & 16.658 & 44.906 \\
\hline Eslovenia & 3.428 & 476 & 3.190 & 7.094 \\
\hline Total EU-15 & 3.874 .765 & 325.844 & 8.605 .750 & 12.806 .379 \\
\hline Total nuevos miembros & 673.629 & 36.788 & 611.338 & 1.321 .755 \\
\hline TOTAL UE-27 & 4.548 .394 & 362.632 & 9.217 .088 & 14.128 .134 \\
\hline
\end{tabular}

Tabla 2. Empleo remunerado en la Economía Social. Unión Europea (2009-2010).

Fuente: Monzón y Chaves (2012). 
que tienen carencias y por lo tanto dificultades para acceder a los bienes y servicios considerados básicos para el bienestar social; pero también se accede, desde la práctica profesional, contribuyendo en la tarea de convertir una necesidad, una carencia en un problema social, objeto de atención por parte de las políticas sociales.

Coincidiendo con Payne (1992), consideramos que el Trabajo Social es una disciplina y una actividad socialmente construida, que varía según los momentos y los contextos sociohistórico, aunque su epistemología y ética se mantienen, aportando identidad y cohesión, por encima de los avatares de cada momento y de las circunstancias locales.

Podemos identificar claramente Trabajo Social como la profesión encargada de ayudar a las personas a tomar conciencia de sus necesidades y de sus capacidades personales, de manera que las puedan utilizar para lograr sus objetivos; esto es, promover la autonomía personal mediante la orientación entendida como el proceso de ayuda para tomar decisiones, al tiempo que interviene en el medio en que se encuentra para promover la integración social efectiva. Esta interacción con el medio significa también intervenir en los asuntos colectivos, por lo tanto en los niveles familiar y grupal.

A todo ello se añade que Trabajo Social se sitúa en la supervisión de los desajustes sociales, como una decisión consciente, organizada y dirigida con la finalidad de actuar sobre el medio social y la persona para mejorar o transformar una situación. Una forma de identificar esta relación se concreta, a través de la propuesta que efectúa Natividad de la Red, en que el Trabajo Social contribuye a dar respuesta a las necesidades sociales, a hacer efectivos los derechos, mediante la acción orientada en las siguientes líneas de acción:

a) Evidenciar las necesidades, mediante la recogida de datos, selección de indicadores, de esta forma se aproxima al usuario-ciudadano a los centros de decisión de la política social y se participa en el desarrollo de metodología y protocoles que permiten responder a las necesidades.

b) Definir estándares de cobertura de necesidades básicas, facilitando la liberación de las personas de la dependencia, escasez, debilidad y de la exclusión social.
Conviene destacar que en un Estado de derecho el éxito de la política social pasa por el acceso de todos los ciudadanos a los recursos considerados básicos, y precisamente el Trabajo Social es un eje fundamental de ese proceso. Según se concreta en el Libro Blanco de Trabajo Social, elaborado en el año 2004, las competencias profesionales son cinco:

- Capacidad para trabajar y valorar de manera conjunta con personas, familias, grupos, organizaciones y comunidades sus necesidades y circunstancias.

- Planificar, implementar, revisar y evaluar la práctica del Trabajo Social con personas, familias, grupos, organizaciones y comunidades y con otros profesionales.

- Apoyar a las personas para que sean capaces de manifestar las necesidades, puntos de vista y circunstancias.

- Actuar para la resolución de situaciones de riesgo con los sistemas cliente, así como para las propias y las de los colegas de profesión.

- Administrar y ser responsables, con supervisión y apoyo, de la propia práctica dentro de la organización.

Si partimos de que, el Trabajo Social es una disciplina que concibe al ser humano en un contexto determinado en interacción con su medio social, cuyo objetivo general es dar respuesta a sus necesidades favoreciendo una conexión entre la persona y su entorno social e institucional, deriva que sus objetivos — según la Conferencia de Directores de Escuelas de Trabajo Social (2002) — son los siguientes:

- Ayudar a disminuir la desigualdad e injusticia social, facilitando la integración social de los grupos de personas marginadas, excluidas socialmente, desfavorecidas económicamente, vulnerables y en situación de riesgo.

- Contribuir a que las personas, los grupos, las organizaciones y comunidades desarrollen destrezas personales e interpersonales que aumenten su poder para enfrentarse a las fuerzas sociales que inciden en su marginación.

- Asistir y movilizar a los individuos, las familias, los grupos, las organizaciones y las comunidades con el fin de mejorar su bienestar y su capacidad para resolver sus problemas. 
- Dar a conocer las oportunidades que tienen los grupos sociales a su disposición, motivarles para tener acceso a esas oportunidades y desarrollar las respuestas emocionales, intelectuales y sociales necesarias para permitirles aprovechar esas oportunidades sin que tengan que renunciar a sus rasgos personales, culturales y de origen.

Entre las diversas áreas profesionales en las que en las que los trabajadores y trabajadoras sociales realizan y habrán de realizar su actividad profesional, la Conferencia destacó las funciones de: asistencia, prevención, promoción y educación, mediación y arbitraje, rehabilitación, planificación, gerencia y administración, investigación y docencia.

Una vez efectuada esta aproximación a las funciones desempeñadas por Trabajo Social, procede ahora identificar las funciones propias del Sistema Público de Servicios Sociales, descritas en los distintos textos legislativos, encontrar puntos de encuentro y coincidencia y contrastar a continuación si se corresponden con las primeras. Con la finalidad de no realizar un abordaje exhaustivo, hemos optado por extraer una muestra que consiste en el análisis de las tres últimas leyes autonómicas promulgadas, de manera que nos permita observar la tendencia actual y la forma en la que se plasma en estos textos de segunda y tercera generación el ámbito competencial del sistema. Las comunidades autónomas elegidas son: Castilla-La Mancha, Castilla y León y Aragón. Sus respectivas normas y referencias a las funciones son las siguientes:

- Ley 14/2010 de 16 de diciembre, de ervicios Sociales de Castilla-La Mancha. Artículo 5. Objetivos del Sistema Público de Servicios Sociaes.

- Ley 16/2010, de 20 de diciembre, de Servicios Sociales de Castilla y León, Artículo 1. Los Equipos de Acción Social Básica.

- Ley 5/2009 de 30 de junio, de Servicios Sociales, de Aragón, Artículo 17. Funciones de los servicios sociales especializados.

Se puede contrastar que las leyes más recientes mantienen un nivel de descripción de funciones propias en los dos niveles de intervención y también en los objetivos del siste- ma, absolutamente vinculadas e identificadas con el desempeño profesional del Trabajo Social.

Finalizamos con el estudio de la presencia que las nuevas leyes otorgan a la economía social, concebida en este campo como iniciativa social, como elemento relevante del sistema y, por lo tanto, con posibilidades desempeñar un importante papel en el futuro de los Servicios Sociales, por medio de la participación en el desarrollo de los distintos servicios y prestaciones. El resultado obtenido ha sido el siguiente:

La Ley 14/2010 de 16 de diciembre, de Servicios Sociales de Castilla-La Mancha, artículo 42, se refiere a la concertación con la iniciativa privada:

1. Las administraciones públicas competentes en materia de servicios sociales podrán encomendar a entidades privadas, prioritariamente las de la iniciativa social, la provisión o gestión de prestaciones previstas en el catálogo mediante concierto, convenio o contrato, ajustándose la pertinencia de su aplicación al carácter de la actividad a contratar o a la provisión de servicios de que se trate, siempre que tales entidades cuenten con la oportuna autorización y figuren inscritas en el Registro de Entidades y Centros de Servicios Sociales.

La Ley 5/2009 de 30 de junio, de Servicios Sociales de Aragón, en su artículo 84, relativo al fomento de la iniciativa social:

Los poderes públicos promoverán la colaboración de las entidades sin ánimo de lucro en la realización de actividades de servicios sociales que se ajusten a la política definida por la planificación estratégica de servicios sociales o los objetivos marcados por la normativa aplicable en cada caso.

La Ley 16/2010, de 20 de diciembre, de Servicios Sociales de Castilla y León, en su artículo 87, relativo al fomento de la iniciativa social sin ánimo de lucro:

1. Las administraciones públicas de Castilla y León fomentarán la creación y desarrollo de entidades de iniciativa social sin ánimo de lucro y las relacionadas con el voluntariado, garanti- 
zando su actuación coordinada en el sistema de servicios sociales de responsabilidad pública según lo definido por la planificación estratégica de servicios sociales o los objetivos marcados por la normativa aplicable en cada caso.

2. Las administraciones públicas de Castilla y León, ante análogas condiciones de calidad, eficacia y costes, darán prioridad a la colaboración con entidades de iniciativa social sin ánimo de lucro.

3. La participación en el sistema de servicios sociales de las entidades de iniciativa social sin ánimo de lucro, como Cruz Roja Española y Cáritas, merecerá una atención especial.

Se constata una presencia de la economía social en esta nueva normativa, recogiendo incluso algunas entidades concretas.

No obstante, en el momento presente resulta difícil realizar un análisis prospectivo ya que, claramente, nos encontramos ante un punto de inflexión en la continuidad de las políticas públicas; estamos en un momento de revisión y «deconstrucción» de todo lo anterior, sobre todo en lo que se refiere a la sostenibilidad del sistema, el futuro pasa por el diseño de modelos híbridos en los que se establecen nuevas alianzas para incorporar nuevos aliados al despliegue de un sistema que requiere esfuerzos para reforzar su eficacia y su eficiencia. El Estado de bienestar diversificado (o híbrido) parece entrar dentro del cuadrante responsabilidad colectiva, máxima descentralización, identificando las aspiraciones colectivas y dándoles respuesta, pero a través del incremento de los agentes participantes en la dispensación. En ese escenario no parece haber duda acerca del incremento del papel de la Tercer Sector en su conjunto. Por lo tanto, a pesar de las incertidumbres, dudas e inquietudes, aparecen grandes posibilidades de confluencia y convergencia desde el ámbito profesional del Trabajo Social y las iniciativas sociales.

\section{Conclusiones}

Más de un 1.200.000 personas trabajaban en las más de 200.000 empresas y entidades de la economía social en España en el año 2008. De ellas algo más de la mitad tenían su puesto de trabajo en aquellas entidades más vinculadas con los servicios sociales y sociosanitarios como: las asociaciones privadas al servicio de los hogares, las fundaciones, las entidades singulares como Cáritas, Cruz Roja y ONCE, las empresas de inserción y los centros especiales de empleo.

A nivel europeo el panorama es análogo: se estima en 2009-2010 más de 14.000.000 personas trabajando en la economía social europea y de ellas más de 9.200.000 las entidades asociativas y otras entidades sin fines de lucro. No solamente se trata de una realidad económica importante, que supone el 6,5 por ciento del empleo remunerado en la Unión Europea, sino también de una realidad dinámica y en crecimiento sostenido desde los años 1990.

Es precisamente en los sectores y tipos de entidades donde mayores tasas de crecimiento se obtienen, como los Servicios Sociales y sociosanitarios, donde existe un mayor potencial de empleo para los trabajadores sociales. A ello se une el cambio en el contexto regulatorio en España en los últimos años, que está confiriendo unas objetivas condiciones para este despliegue laboral.

\section{Referencias bibliográficas}

Conferencia de Directores de Escuelas de Trabajo Social de España. (julio, 2002). Trabajo Social como titulación de grado en el Espacio Europeo de Educación Superior con un perfil propio $y$ diferenciado. Madrid: CRUE.

Consejo Económico y Social. (2008). Informe 3/2008, sobre el borrador del V Plan nacional de acción para la inclusión social, 2008-2010. Madrid: CES.

Defourny, J. y Nyssens, M. (enero, 2011). Approches européennes et américaines de l'entreprise sociale: une perspective comparative. Revue internationale de l'économie sociale, 319, 18-35.

De la Red, N. (2000). La metodología del trabajo social desde el marco de las ciencias sociales. Cuadernos Andaluces de Bienestar Social, 8, 9-32.

Etxezarreta, E. y Bakaikoa, B. (2011). La participación de la Economía Social en el ámbito de la dependencia: una aproximación teórica. CIRIEC-España, Revista de economía pública, social y cooperativa, $71,25-48$. 
Gómez, J.M., Peláez, F.J. y García, A. (2011). Dependencia, envejecimiento demográfico y economía social, CIRIEC-España, Revista de economía pública, social y cooperativa, 71,101-128.

Jiménez, A. (2011). Haciendo de la necesidad virtud: la atención a las personas en situación de dependencia como vector del crecimiento del empleo. CIRIEC-España, revista de economía pública, social y cooperativa, 71, 129-146.

Jiménez, J. y Morales, A.C. (2011). Social economy and the fourth sector, base and protagonist of social innovation, CIRIEC-España, Revista de economía pública, social y cooperativa, 73, 33-60.

Jiménez, A. y Rodríguez, A. (2012). La Economía Social y la atención a la dependencia. (Documento de trabajo 177/2012). Madrid: Fundación Alternativas.

Juste, A., Gómez, J.M. y Fernández, J. (2011). Economía social y desarrollo local/rural, Estudios de economía aplicada, 29 (1), 189-222.

Marshall, T.H. y Bottomore, T. (1998). Ciudadanía y clase social. Madrid: Alianza Editorial.

Martin, S. y Lejarriaga, G. (2011). Las empresas de participación de trabajo asociado ante los desafíos del futuro en un contexto de crisis económica. CIRIEC-España, Revista de economía pública, social y cooperativa, 72, 239-261.

Monzón, J.L. (dir.) (2010). La Economía social en España en el año 2008. Ámbito, magnitudes, actividades y tendencias, Valencia: CIRIEC-España .

Monzón, J.L. y Barea, J. (2007). Manual de orientación para la elaboración de una cuenta satélite de las empresas de la Economía Social. Bruselas: European Commission.

Monzón, J.L. y Chaves, R. (2012). La Economía Social en la Unión Europea. Bruselas: Comité Económico y Social Europeo.

Monzón, J.L. y Marcuello, C. (2012). Economía Social y Empresas Sociales. [Monografía].Ciriec-España, Revista de economía pública, social y cooperativa, 75, 199-221.

Morales, A.C. (2011). Modelos de integración y desarrollo estratégico de las cooperativas sociosanitarias andaluzas. CIRIEC-España, Revista de economía pública, social y cooperativa, 71, 203-226.

Payne, M. (1995). Teorías contemporáneas del Trabajo Social. Una introducción critica. Barcelona: Paidós.

Pastor, E. (2011). El papel de la economía social como motor del cambio social y la democratización sostenible de las políticas públicas sociales en el ámbito local. Revesco - revista de estudios cooperativos, 104, 143-169.

Ramírez, J.M. (2011). Impacto sobre el empleo del sistema de la dependencia. CIRIEC-España, Revista de economía pública, social y cooperativa, 71, 77-100.

Ruiz, O. (2011). La Economía Social y la atención a la dependencia en España: una aproximación a través de los centros de larga estancia. CIRIEC-España, Revista de economía pública, social y cooperativa, 71, 147-174.

Salinas, F., Osorio, L.E. y Albarán, C. (2011). El sector público y la Economía Social en la atención a la dependencia. CIRIEC-España, Revista de economía pública, social y cooperativa, 71, 9-24.

Toia, P. (ponente) (19 de febrero de 2009). Informe sobre Economía Social (Toia Report). Bruselas: Parlamento Europeo.

Toledano, N. (2011). Social entrepreneurship: The new narrative for the practice of the Social Economy, CIRIEC-España. Revista de economía pública, social y cooperativa, 73, 1-31.

Zaragoza, G. (1997). Los Servicios Sociales Municipales en la provincia de Valencia. Luces y sombras de un sistema. Valencia: Reproexpres. 